LA-UR $97-4011$

author(s): P.H. BRADLEY

submitted to: Proceedings of The Fourth

Whole Earth TELESCOPE LUERKSHOP

JULY 21-25, 1997

KONINKI,

QuAthis

Q:STRBUTION ON THE DOCUMENT IS UNLIMTTED 


\section{DISCLAIMER}

This report was prepared as an account of work sponsored by an agency of the United States Government. Neither the United States Government nor any agency thereof, nor any of their employees, makes any warranty, express or implied, or assumes any legal liability or responsibility for the accuracy, completeness, or usefulness of any information, apparatus, product, or process disclosed, or represents that its use would not infringe privately owned rights. Reference herein to any specific commercial product, process, or service by trade name, trademark, manufacturer, or otherwise does not necessarily constitute or imply its endorsement, recommendation, or favoring by the United States Government or any agency thereof. The views and opinions of authors expressed herein do not necessarily state or reflect those of the United States Government or any agency thereof. 
Baltic Astronomy, vol.6, xxx-xxx, 1997.

\title{
ASTEROSEISMOLOGY OF DAV WHITE DWARF STARS
}

Paul A. Bradley

XTA, MS B220, Los Alamos National Laboratory, Los Alamos, NM 87545

Received October 1, 1997.

\begin{abstract}
I review the seismological structural determinations of $\mathrm{ZZ}$ Ceti stars done to date, and supplement these with additional preliminary determinations of my own. I compare the constraints on the hydrogen layer mass to see what trends emerge and also determine if the observed hydrogen layer masses are consistent with proposed theories. I then look ahead to the prospects of further DAV white dwarf seismology.
\end{abstract}

Key words: Stars: White Dwarfs, Stars: interiors, Stars: Oscillations, Stars: individual: G 117-B15A, G 226-29, G 29-38, GD 66, GD 154, GD 165, GD 385, HL Tau 76, HS 0507+0434, L 19-2, PG 2303+242, R 548

\section{Introduction}

The goal of asteroseismology is to determine the structure of stars by comparing the observed pulsation properties with those predicted by stellar models. The DAV (or ZZ Ceti) stars are hydrogen atmosphere white dwarfs that undergo nonradial oscillations. They are otherwise normal DA white dwarfs, so their seismology is crucial for understanding the structural properties of DA white dwarfs in general. Determining the range of hydrogen layer masses in ZZ Ceti stars is particularly important, given the conflict between different hypothesis concerning the hydrogen layer masses of DA white dwarfs and the effect of the hydrogen layer mass on the inferred ages and stellar masses of DA white dwarfs in general.

While DAV star seismology will help our understanding of white dwarfs, there are obstacles to making it reality. Observations of the

Send offprint requests to: P.A. Bradley 
DAV stars show that they tend to cluster in two main groups: a hotter group that has a small number of short period modes present, and a cooler group that tends to have more modes, but stronger nonlinear effects and variable amplitudes.

The hotter DAV stars tend to have only a few, short period modes, making seismology difficult for two reasons. First, without some way to constrain the mode identity of the observed pulsations, one can fit a distressingly large number of models to the observations; the statement "anything can fit" is not seismologically interesting. The second problem is that the small number of observed modes implies fewer constraints on the underlying structure. In practice, the shortest period modes of many hot DAV stars are sufficiently short that we can determine interesting hydrogen layer mass constraints, even if we cannot derive a detailed picture of the interior. The other good news about these stars is that they tend to have stable period spectra and only small amplitude changes. Therefore, a single WET run is likely to find all the modes that are present, and over a long enough period of time one can hope to obtain interesting limits on the rate of period change (Kepler et al. 1995a, b).

The cooler DAV stars have a tempting number of peaks present, but presently this wealth is superficial. The typical cool DAV frequency spectrum has several large amplitude peaks $\left(f_{i}\right)$, and these generate harmonics $\left(n f_{i}\right)$, frequency $\operatorname{sum}\left(f_{i}+f_{j}\right)$, and frequency difference $\left(f_{i}-f_{j}\right)$ peaks. Thus, the first difficulty is extracting the real modes that we are interested in for seismology from the nonlinear interaction peaks. The cooler DAVs also tend to have modes with considerable amplitude variation, so that the two-week "snapshot" a WET run produces only reveals part of the possible modes in the modal spectrum. Extending the time baseline with supplementary (single-site) data shows that over time, additional modes will appear, and modes that reappear do so in the same place (Kleinman 1995). The above evidence suggests that once deciphered, the cool DAVs will be richer targets for seismology.

\section{Why is DAV Seismology Critical?}

The difficulty of extracting enough observed periods from the DAVs to be seismologically interesting discouraged any serious attempts at their seismology until recently. This started to change with Fontaine et al.'s paper on G 226-29 (1992) and now the seismology business is making some progress. Part of this stems from the 
fact that the models are now good enough (Wood 1992, 1994, 1995; Brassard \& Fontaine 1994; Bradley 1996) that the periods are accurate enough for quantitative comparisons to observations. Another reason is that some DAV stars have short enough minimum periods to limit the number of possible mode identifications, and allow one to derive interesting hydrogen layer mass constraints. Finally, DA white dwarfs are the most numerous class of white dwarf, which provides extra impetus for determining as much as possible about their internal structure. I'm pleased to say that experience suggests that seismology is not as difficult as once feared, though caution is still mandatory.

DAV seismology is crucial, and one reason is the number fraction of DA to non-DA white dwarfs is a strong function of effective temperature (see Kepler \& Bradley 1995 and Fontaine \& Wesemael 1997 for reviews). Here, I highlight some of the observational puzzles, with the note that the spectroscopic evidence now seems to point to a range of hydrogen layer masses, rather than "all thick" or "all thin". The first critical piece of evidence is the existence of the "DB Gap". We see DO white dwarfs above 45,000 K, but between 45,000 and $30,000 \mathrm{~K}$ we see only DA white dwarfs (the so-called "DB Gap"), which implies that the hottest helium-rich white dwarfs must transform themselves to DA white dwarfs. Vennes et al. (1997) studied the hot DA white dwarfs observed by the EUVE satellite and show that if one wants to match the mean stellar mass of this hot white dwarf sample to the cooler white dwarf sample of Bergeron, Saffer, \& Liebert (1992), then the hydrogen layer mass must be considerably less than $10^{-4} \mathrm{M}_{\star}$. In this context, $\mathrm{M}_{\mathrm{H}} \leq 10^{-6} \mathrm{M}_{\star}$ suffices, and this is not surprising, given that the two major channels of spectral evolution will have DA stars through the 30,000 to $45,000 \mathrm{~K}$ temperature range. Down at about $10,000 \mathrm{~K}$, Bergeron et al. (1990, see also Bergeron, Ruiz, \& Leggett 1997) shows there is an additional source of pressure in DA white dwarf hydrogen lines. This can be explained either by a sudden increase in $\log g$ (stellar mass) or if spectroscopically unobservable helium is being "dredged up" from below by the surface convection zone. If helium is being "dredged up" by the convection zone starting at about $10,000 \mathrm{~K}$, it implies the hydrogen layer mass must be about $10^{-10} \mathrm{M}_{\star}$ or thicker. However, "dredging up" does not demand that the resultant atmosphere be pure helium (i.e., completely mixed). Also, some DA white dwarfs must have hydrogen layer masses closer to $10^{-6} \mathrm{M}_{\star}$ for convective mixing to be delayed until about 5,000 K. For further details on the 
spectral evolution of the coolest white dwarfs, I refer the reader to Bergeron, Ruiz, \& Leggett (1997).

In response to the observations, several theories appeared. The dominant theories talk about "channels" for white dwarf formation, with the most discussed channels being "born DA" white dwarfs resulting from hydrogen-rich planetary nebula nuclei (PNN) and white dwarfs resulting from the $\mathrm{He} / \mathrm{C} / \mathrm{O}$ PNN that become PG 1159 stars before becoming white dwarfs. Fontaine \& Wesemael $(1987,1991)$ originally suggested that a majority of white dwarfs go through the $\mathrm{He} / \mathrm{C} / \mathrm{O} \mathrm{PNN}$ and PG 1159 phase. Even during the PNN and PG1159 phase, these white dwarfs have at least some hydrogen, but it is spectroscopically undetectable as the star evolves through the PG 1159 region. As the PG 1159 spectral type star cools, the hydrogen diffuses to the surface and all of these stars have sufficient hydrogen diffused to the photosphere by $45,000 \mathrm{~K}$ so that no white dwarf has helium present, which explains the "DB gap". Other DA white dwarfs would still originate with the hydrogen-rich PNN and would have hydrogen layer masses of $10^{-4} \mathrm{M}_{\star}$ minus whatever the PNN wind stripped off. Fontaine \& Wesemael (1997) discuss the changes in the observational and theoretical evidence for the extremes of "thick" versus "thin" hydrogen layer hypotheses, and suggest that the available evidence supports a range of hydrogen layer masses between $\sim 10^{-10} \mathrm{M}_{\star}$ and $\sim 10^{-4} \mathrm{M}_{\star}$. These explanation of these two channels and their spectral evolution are still qualitatively correct, but recent observations suggest that the $\mathrm{He} / \mathrm{C} / \mathrm{O}$ PNN through PG1159 star channel is not the dominant one. These theories neglect contributions from other minor "channels", such as extreme horizontal-branch stars and interacting binary white dwarfs.

On the other extreme, Clemens (1994) examined the pulsation spectra of the hotter DAV white dwarfs as a class and demonstrated that they have modes with remarkably similar period structure. When one allows for differences due to effective temperatures and stellar mass, it appears as if all DAVs select modes from a single period spectrum template, which implies a narrow range of hydrogen mass for all the DAV stars or a miraculous coincidence. Comparing the derived period template to the then extant models, Clemens derived a hydrogen layer mass near $10^{-4} \mathrm{M}_{\star}$. However, there is the underlying assumption that the modal structure, and hence the composition profile is the same for all the ZZ Ceti stars. Nature does not require this, and I consider it vitally important to independently 
determine at least the $\ell$ identity of the ZZ Ceti star modes to put Clemens' assertion on firmer ground or refute it.

The different pictures painted by these theories means that we need a way to figure out the hydrogen layer mass from seismology, without relying solely on Clemens's (1994) work and assumptions about the mode identity yielding a narrow range of hydrogen layer masses. To do this, one has to adopt an exhaustive approach by comparing the observed periods to an extensive grid of models. It is relatively easy to find a model that fits - just ask any theorist - the hard part is finding all the possible fits and ruling out any other possibility, especially since we don't necessarily know the mode identity of a given observed period.

\section{Observational Constraints on Identification of Modes}

Seeing a large number of modes in a pulsating white dwarf begins the struggle for seismology of the star; the pivotal problem is determining the $\ell$ and $k$ value of each mode. I illustrate this problem with examples based on G 117-B15A and L 19-2.

For G 117-B15A, Robinson et al. (1995) and Fontaine et al. (1996) prove that the dominant 215 s mode is $\ell=1$, but do not constrain the $k$ value, although theoretical models show it must be either $k=1$ or 2 . The vexing point is the $\ell$ identification of the 270 and $304 \mathrm{~s}$ modes, along with the overtone number. The simplest interpretation is that all are $\ell=1$ modes, and this identification has indirect support from the work of Brassard et al. (1993). For seismological purposes, the $\ell=1$ nature of the $215 \mathrm{~s}$ mode provides the strongest constraint on the hydrogen layer mass, while other modes help refine the structural determination. Geometric cancellation effects (Dziembowski 1977) favor our seeing the lowest possible $\ell$ modes, and $\ell=1$ modes dominate the power spectra of the pre-white dwarf GW Virginis stars and the DB white dwarf GD 358 (Winget et al. 1994). In the case of L 19-2 (O'Donoghue \& Warner 1982, 1987) and GD 165 (Bergeron et al. 1993), we have ZZ Ceti stars with two different $\ell$ values, and the largest amplitude modes are $\ell=1$. Although this evidence is circumstantial, the above discussion suggests that $\ell=1$ modes are favored, and in no case do we have a white dwarf with a dominant $\ell=2$ mode. This suggests that we are seeing only $\ell=1$ modes in $\mathrm{G} 117-\mathrm{B} 15 \mathrm{~A}$.

As I just mentioned, L 19-2 has modes of different $\ell$ excited, because the two multiplets at 113 and $118 \mathrm{~s}$ are too closely spaced to 
reasonably be consecutive overtones of the same $\ell$, and furthermore, the multiplet structure is different. As Bradley (1993) points out the different frequency splitting of the 113 and $118 \mathrm{~s}$ is consistent with the $113 \mathrm{~s}$ doublet being two of the five $\ell=2$ members (with $\Delta m=2$ ) and the $118 \mathrm{~s}$ mode being 2 of the 3 members of an $\ell=$ 1 triplet. Continuing with the frequency splitting trends, Bradley (1993) identifies the 118, 192, and 350 s modes as $\ell=1$, while the 113 and $143 \mathrm{~s}$ modes should be $\ell=2$. Sullivan (1995) confirms the formerly speculative identification for the $143 \mathrm{~s}$ mode by revealing four of the five multiplet members, all with splittings near $18.5 \mu \mathrm{Hz}$. The $118 \mathrm{~s}$ mode is short enough that it must be the $\ell=1, k=1$ mode. This is actually enough to constrain the hydrogen layer mass to lie near $10^{-4} \mathrm{M}_{\star}$; the other modes mainly serve to constrain the core structure, stellar mass, and helium layer mass better.

\section{Modeling Strategy}

Bradley (1997) describes the seismology of G 117-B15A and $R 548$ in considerable detail, and the next two sections illustrate the considerable search through parameter space one must make in order to determine which models do not fit the observed periods, as well as those that do fit. We will not repeat this discussion, but rather describe by way of example the process of how to choose starting points for seismology and refining the model fits. We use G $117-$ $\mathrm{B} 15 \mathrm{~A}$ as the example, because of the $\ell$ constraint on the $215 \mathrm{~s}$ mode.

The first constraints for seismology are the effective temperature and the stellar mass as estimated from the surface gravity. Bergeron et al. (1995) is the best reference here, both for completeness and uniformity of analysis. Unfortunately, Koest \& Vauclair (1997) show that the real error bars on the temper. ire and gravity are distressingly large because of the behavior of the hydrogen line profiles near $12,000 \mathrm{~K}$. Next, we need at least an initial constraint on the $\ell$ and $k$ identification of the observed modes.

As mentioned previously, Robinson et al. (1995) and Fontaine et al. (1996) show the $215 \mathrm{~s}$ mode is $\ell=1$, and this is short enough that it can only be the $k=1$ or $k=2$ mode; the hydrogen layer mass is then about $10^{-7} \mathrm{M}_{\star}$ or $10^{-4} \mathrm{M}_{\star}$ respectively. G $117-\mathrm{B} 15 \mathrm{~A}$ has two additional modes at 270 and $304 \mathrm{~s}$, which Clemens (1994) suggests fit a common mode pattern for the hotter white dwarfs as $\ell=1$ modes. Brassard et al. 1993, Fontaine \& Brassard (1994) and Bradley (1996) show that these three modes can be satisfactorily fit 
as three consecutive overtone $\ell=1$ modes, although Bradley (1993) discusses model fits involving $\ell=2$ modes.

To go further, we have to examine the period trends of the modes corresponding to the 270 and 304 s periods when we change various structural parameters one at a time and keep the stellar mass fixed. Bradley $(1996,1997)$ presents the results of this parameter search. For G $117-\mathrm{B} 15 \mathrm{~A}$, the $270 \mathrm{~s}$ mode period is most sensitive to refinement in the hydrogen layer mass. By contrast, the $304 \mathrm{~s}$ mode is strongly dependent on the extent of the $\mathrm{C} / \mathrm{O}$ core. The independent behavior of these two modes means that we can obtain a good initial seismological fit by tuning the fit each mode almost independent of the others. In outline form, the procedure is:

1. Pick a stellar mass and effective temperature consistent with observations.

2. Use a "standard" post-Asymptotic Giant Branch star helium layer mass of $10^{-2} \mathrm{M}_{\star}$ and a "nominal" $\mathrm{C} / \mathrm{O}$ profile (see below).

3. Adjust the hydrogen layer mass to obtain an $\ell=1, k=1$ or 2 mode near $215 \mathrm{~s}$.

4. Adjust the temperature and hydrogen layer mass slightly to bring the $270 \mathrm{~s}$ period into agreement.

5. Adjust the $\mathrm{C} / \mathrm{O}$ profile to make the $304 \mathrm{~s}$ mode agree with observations.

6. Refine the fit further and/or perform sensitivity studies by changing the hydrogen layer mass, helium layer mass, and core structure.

To decide which model fits the observed periods the best, we simply average the absolute value of the difference between the observed and theoretical periods, and denote this average by $\Delta$ in the period comparison tables.

\section{A Model Fitting Example for G 117-B15A}

For G 117-B15A, we use Bergeron et al.'s effective temperature of $11,600 \mathrm{~K}$ and $\log g=7.97$, which implies a mass of $0.59 \mathrm{M}_{\odot} \cdot \mathrm{Be}-$ cause we already have an extensive model grid for $0.60 \mathrm{M}_{\odot}$, we use this mass and attempt to match the three observed periods, assuming that the $215 \mathrm{~s}$ mode is $k=1$.

First, we look at plots (or tables) of $\ell=1$ periods as a function of hydrogen layer mass, looking for where the $215 \mathrm{~s}$ period is well matched for $k=1$ at temperatures near $11,600 \mathrm{~K}$. This occurs when the hydrogen layer mass is near $10^{-7} \mathrm{M}_{\star}$ (see Table 1 ). At this point, 
Table 1.0.60M $\odot$ models versus G 117-B15A: Vary H Layer mass

$\begin{array}{ccccccc}\mathrm{M}_{\mathrm{H}} / \mathrm{M}_{\star} & \mathrm{T}_{\text {eff }} & (1,1) & \Delta P & (1,2) & (1,3) & \Delta \\ & (\mathrm{K}) & (\mathrm{s}) & (\mathrm{s}) & (\mathrm{s}) & (\mathrm{s}) & (\mathrm{s}) \\ \mathrm{G} 117-\mathrm{B} 15 \mathrm{~A} & 11,620 & 215.2 & 55.8 & 271.0 & 304.4 & - \\ 1.0 \times 10^{-7} \mathrm{M}_{\star} & 11,560 & 213.5 & 62.8 & 276.3 & 293.8 & 5.9 \\ 2.0 \times 10^{-7} \mathrm{M}_{\star} & 11,590 & 213.2 & 54.8 & 268.0 & 286.2 & 7.7 \\ 2.0 \times 10^{-7} \mathrm{M}_{\star} 11,090 & 218.2 & 53.6 & 271.8 & 291.7 & 5.3 \\ 3.0 \times 10^{-7} \mathrm{M}_{\star} 11,590 & 213.1 & 46.5 & 259.6 & 284.5 & 7.7\end{array}$

we also consider the $270 \mathrm{~s}$ mode, and the correct period spacing of $55.8 \mathrm{~s}$ occurs only when the hydrogen layer mass is near $2 \times 10^{-7} \mathrm{M}_{\star}$. The period spacing $\Delta P$ is more relevant, because the relatively coarse temperature grid used in the initial sweep of evolutionary models does not guarantee the $k=1$ mode will be at $215.2 \mathrm{~s}$.

At this point, the first two modes fit, but the $k=3$ periods are too short for the "nominal" $\mathrm{C} / \mathrm{O}$ core structure used here. The "nominal" $\mathrm{C} / \mathrm{O}$ core structure has $20 \%$ carbon out to $0.75 \mathrm{M}_{\star}$, whereupon we impose a linear ramp to pure carbon by $0.90 \mathrm{M}_{\star}$. This profile captures the essence of the more complicated $\mathrm{C} / \mathrm{O}$ profiles of Mazzitelli \& D'Antona (1986a, b). When we change the core structure, we vary only two parameters. First, we change the amount of carbon in the core, but leave the linear ramp boundaries intact. Alternatively, we can fix the core carbon abundance at $20 \%$ and move the inner boundary point of the linear ramp from the default of $0.75 \mathrm{M}_{\star}$. Other possibilities exist, but these two parameters capture most of the possible effects on the theoretical periods for a minimum number of additional free parameters.

With this said about the core structure of the models, we fix the hydrogen layer mass at $2 \times 10^{-7} \mathrm{M}_{\star}$ and vary the core structure (see Table 2). A model with the inner transition point moved out to $0.85 \mathrm{M}_{\star}$ provides a good fit to all the periods and the model temperature is within $100 \mathrm{~K}$ of the spectroscopic temperature. Alternatively, a $50: 50 \mathrm{C} / \mathrm{O}$ core out to $0.75 \mathrm{M}_{\star}$ at $11,460 \mathrm{~K}$ fits the three periods to an average of better than $1 \mathrm{~s}$. At this point, both models are equally plausible representations for the structure of G 117-B15A, and they say that the core is at least $50 \%$ oxygen, consistent with the oxygen mass fraction derived by the evolutionary models of Mazzitelli \& D'Antona (1986a, b) and Salaris et al. (1997). The seismologically predicted $\mathrm{C} / \mathrm{O}$ transition region may be a bit further in than 
Table 2.0.60M $\odot$ models versus $\mathrm{G} 117-\mathrm{B} 15 \mathrm{~A}(215 \mathrm{~s}$ is $k=1)$. Fits as a function of core mass fraction and extent for $M_{H}=2 \times$ $10^{-7} \mathrm{M}_{\star}$.

$\begin{array}{ccccccc}\text { Fraction C/O } & \mathrm{M}_{c / o} / \mathrm{M}_{\star} & \begin{array}{c}\mathrm{T}_{\text {eff }} \\ (\mathrm{K})\end{array} & \begin{array}{c}(1,1) \\ (\mathrm{s})\end{array} & \begin{array}{c}(1,2) \\ (\mathrm{s})\end{array} & \begin{array}{c}(1,3) \\ (\mathrm{s})\end{array} & \begin{array}{c}\text { (s) } \\ \text { G 117-B15A }\end{array} \\ & & 11,620 & 215.2 & 271.0 & 304.4 & - \\ 20: 80 & 0.85 & 11,510 & 214.7 & 269.2 & 301.7 & 1.7 \\ 20: 80 & 0.85 & 11,430 & 215.6 & 269.9 & 302.9 & 1.0 \\ 20: 80 & 0.80 & 11,560 & 213.8 & 268.5 & 293.1 & 4.7 \\ 20: 80 & 0.75 & 11,590 & 213.2 & 268.0 & 286.2 & 7.7 \\ 20: 80 & 0.75 & 11,590 & 213.2 & 268.0 & 286.2 & 7.7 \\ 50: 50 & 0.75 & 11,460 & 217.3 & 270.9 & 304.3 & 0.8 \\ 80: 20 & 0.75 & 11,990 & 213.4 & 268.5 & 306.0 & 2.0 \\ 80: 20 & 0.75 & 11,560 & 217.4 & 271.5 & 311.2 & 3.3\end{array}$

the above evolutionary calculations suggest, but the core/envelope construction of the models does not allow us to put $\mathrm{C} / \mathrm{O}$ transition regions past about $0.90 \mathrm{M}_{\star}$.

Having found a set of "best fitting" models at $0.60 \mathrm{M}_{\odot}$ when the $215 \mathrm{~s}$ mode is $k=1$, we would now assume the $215 \mathrm{~s}$ mode is $k=2$ and use the same procudure to determine the structure of the best fitting model(s). Here, the best fitting hydrogen layer mass is about $8 \times 10^{-5} \mathrm{M}_{\star}$ the helium layer mass is about $10^{-2} \mathrm{M}_{\star}$. Again, there are two equally possible $\mathrm{C} / \mathrm{O}$ core profiles. One $\mathrm{C} / \mathrm{O}$ core possibility has the inner transition point at $0.83 \mathrm{M}_{\star}$ and a $20: 80 \mathrm{C} / \mathrm{O}$ core, while the other possible core profile has a $50: 50 \mathrm{C} / \mathrm{O}$ core out to $0.75 \mathrm{M}_{\star}$. Both profiles fit all three periods to within $2.5 \mathrm{~s}$ or better.

Finally, to bracket the observed uncertainties in $T_{\text {eff }}$ and $\log g$, we examine models at the extreme spectroscopic masses, which are 0.55 and $0.65 \mathrm{M}_{\odot}$. Again, we consider the possibility of the $215 \mathrm{~s}$ being $k=1$ or $k=2$. We derive hydrogen layer masses of $1 \times 10^{-6} M_{\star}$ or $1.5 \times 10^{-4} \mathrm{M}_{\star}$ for $0.55 \mathrm{M}_{\odot}$ models, and values of $5 \times 10^{-8} \mathrm{M}_{\star}$ or $3 \times 10^{-5} \mathrm{M}_{\star}$ for $0.65 \mathrm{M}_{\odot}$ models. In all cases, the core is oxygen-rich and the core composition profiles are similar to the $0.60 \mathrm{M}_{\odot}$ profiles. 


\section{Seismological Structure Determinations for the ZZ Ceti Stars}

In this section, I summarize the available structural determinations of ZZ Ceti stars in the literature. Several more ZZ Ceti stars have enough period information for seismology, and I present preliminary estimates of their structure here.

\section{$6.1 G 226-29$}

This star has a triplet of modes at $109 \mathrm{~s}$ (Kepler et al. 1995c), which Kepler et al. (1995d) show is $\ell=1$, although one member of the multiplet is better fit as an $\ell=2$ mode. Fontaine et al. (1992) started the current efforts of DAV white dwarf seismology with this star, and obtained a hydrogen layer mass of about $2 \times 10^{-4} \mathrm{M}_{\star}$ for an $\ell=1, k=1$ mode. More recently, I redid the analysis in Kepler et al. (1995c) using the effective temperature of $12,460 \mathrm{~K}$ and $\log g$ value of 8.21 from Bergeron et al. (1995). In addition, I the model should have a parallax close to the trigonometric parallax, which HIPPARCOS gives as 91.1 mas (Vauclair et al. 1997). With these constraints, I derive a hydrogen layer mass of $\approx 10^{-4} \mathrm{M}_{\star}$, a mass of $0.80 \mathrm{M}_{\odot}$, and the seismological parallax is $95.7 \mathrm{mas}^{1}$.

The $\ell=1$ identity and a mass of $\sim 0.80 \mathrm{M}_{\odot}$ means the hydrogen layer of G 226-29 must be "thick". Also, the $k=1$ identity is secure, because higher $k$ values would imply unreasonably thick hydrogen layers.

\section{$6.2 R 548$ ( $\equiv Z Z$ Ceti)}

Bradley (1997) also examined R 548, and determined much better constraints on the structure, because the two observed modes are doublets. Presumably these are two highest amplitude peaks of two $\ell=1$ triplets split by slow rotation. Using the splitting information, in addition to spectroscopic and period constraints, leads to a best fitting model with a mass of $0.54 \mathrm{M}_{\odot}$ and a hydrogen layer mass of $1.5 \times 10^{-4} \mathrm{M}_{\star}$ because the $213 \mathrm{~s}$ mode is $k=2$. Models with

1 Before HIPPARCOS, my work led me to believe the ground based parallax (81.9 mas) was too small, and I predicted that HIPPARCOS would find a parallax angle closer to 95 mas (Turon \& van Leeuwen 1995). It is. 
a hydrogen layer mass near $10^{-7} \mathrm{M}_{\star}$ with a $k=1213 \mathrm{~s}$ mode fail to match the observed fine structure pattern although they can fit the observed periods. This star most likely has a "thick" hydrogen layer, and the seismological work of Bradley (1997) favors the $213 \mathrm{~s}$ multiplet being $k=1$.

R 548 has a large parallax discrepancy; Bradley (1997) predicts a parallax angle of about 29 mas, while Van Altena et al. (1994) quote a value of $14.9 \pm 2.0$ mas. Bradley (1997) discusses this discrepancy in some detail, and the discrepancy cannot be the result of an undiscovered binary. It appears that the ground based parallax value must have larger true errors than quoted.

\subsection{G $117-B 15 A$}

Bradley (1997) presents a detailed analysis of possible models that fit all three periods and the spectroscopic constraints. The best fitting hydrogen layer mass has two possible values, depending on whether the $215 \mathrm{~s}$ mode is $k=1$ or $k=2$. The best fitting stellar mass is close to $0.60 \mathrm{M}_{\odot}$ in both cases, and the hydrogen layer mass is close to $10^{-4} \mathrm{M}_{\star}(k=2)$ or $10^{-7} \mathrm{M}_{\star}(k=1)$. In my nomenclature, the hydrogen layer is either "thick" or "thin". The period structure alone cannot distinguish between $k=1$ or 2 , and we must find fine structure splitting (like in G $226-29$ or R 548) or find additional modes (preferably shorter period) to make a distinction. Fontaine \& Brassard (1994) describe an independent seismological analysis of G $117-\mathrm{B} 15 \mathrm{~A}$. They assume the $215 \mathrm{~s}$ mode is $k=1$ and quote a hydrogen layer mass of $1.3 \times 10^{-6} \mathrm{M}_{\star}$ and a helium layer mass of $2.5 \times 10^{-3} \mathrm{M}_{\star}$. Their hydrogen layer is about 7 times thicker than Bradley's value, while the helium layer is four times thinner. Until we can find the origin of the discrepancy, the independent analyses suggest that the hydrogen and helium layer masses may only be accurate to within a factor of ten.

Finally, G 117-B15A has a large parallax discrepancy; Bradley's models predict a parallax angle of $\sim 17.5$ mas, compared to the Van Altena et al. (1994) value of $10.5 \pm 4.2$ mas. Again, the luminosity implied by seismology and spectroscopy are agree with each other, so my suspicion is the ground-based parallax has an underestimated error. 


\subsection{L $19-2$}

I am currently working on seismological fits for L 19-2, based on Bradley's (1993) mode identification. This star is one of the few DAV white dwarfs that has observable $\ell=2$ modes. I have not exhaustively mapped out parameter space yet, but the most promising models have masses between 0.70 and $0.75 \mathrm{M}_{\odot}$, consistent with the spectroscopic mass. L $19-2$ has the $\ell=1, k=1$ mode near $118 \mathrm{~s}$, which is short enough to require a "thick" hydrogen layer near $10^{-4} \mathrm{M}_{\star}$. The models predict rotational splittings with the right trends, but do not yet agree in detail. In this case, the seismological parallax (42 to 46 mas) agrees perfectly with the ground-based parallax of $44 \pm 5$ mas.

\section{$6.5 G D 165$}

Bergeron et al. (1993) present the period spectrum of this star and use the $120 \mathrm{~s}$ mode as $k=1$ to constrain the hydrogen layer mass at $\sim 10^{-4} \mathrm{M}_{\star}$ for $\ell=1$ or $\sim 10^{-7} \mathrm{M}_{\star}$ for $\ell=2$. There is also a mode near $114 \mathrm{~s}$, which is likely an $\ell=2$ mode; it is too close to the $120 \mathrm{~s}$ mode for both to be the same $\ell$. The period spectrum is quite similar to that of L 19-2, and I suspect the structure is similar, but preliminary work suggests a stellar mass about 0.01 to $0.02 \mathrm{M}_{\odot}$ less. Given a $120 \mathrm{~s} \ell=1$ mode, the hydrogen layer mass is near $10^{-4} \mathrm{M}_{\text {* }}$ and this star has a "thick" hydrogen layer.

GD 165 has a mode near $250 \mathrm{~s}$, which I interpret as the $\ell=1$ analogue of the $143 \mathrm{~s} \ell=2, k=4$ mode in L 19-2. It is not possible to obtain a model that fits the $143 \mathrm{~s}$ period of $\mathrm{L} 19-2$ and at the same time predicts an $\ell=1, k=4$ mode at $250 \mathrm{~s}$. Further seismological modeling work shows that GD 165 must have a different core structure than L 19-2. Like L 19-2, GD 165 has a parallax angle ( 28 to 33 mas) that is consistent with the ground-based parallax of $27.8 \pm 3.4$ mas.

\subsection{G 29-38}

Preliminary work by Bradley \& Kleinman (1997) shows that a $0.75 \mathrm{M}_{\odot}$ model with $\mathrm{M}_{\mathrm{H}}=5 \times 10^{-7} \mathrm{M}_{\star}$ fits 14 of the 16 known modes to within $1 \%$ or $10 \mathrm{~s}$. Based on this model, I predict a parallax angle of 66 mas, smaller than the ground-based parallax of $73.4 \pm$ 4.0 mas, which highlights the well-known discrepancy between the 
parallax mass $\sim 0.8 \mathrm{M}_{\odot}$ and the spectroscopic mass of $\sim 0.7 \mathrm{M}_{\odot}$. The abundance of modes makes me choose the 110 s mode as $\ell=2, k=1$. Thus, this star has a "thin" hydrogen layer. I caution, however, that this seismological determination is critically dependent on the $110 \mathrm{~s}$ mode being $\ell=2, k=1$. If the 110 s mode is say, the $\ell=1, k=1$ mode, then we would be forced towards a stellar mass and hydrogen layer mass similar to that of G 226-29.

\subsection{GD 154}

Pfeiffer et al. (1996) report a favored hydrogen layer of $\sim 2 \times$ $10^{-10} \mathrm{M}_{\star}$, but they cannot rule out thicker hydrogen layer masses. This assumes a stellar mass of $0.70 \mathrm{M}_{\odot}$. I predict a parallax angle of 40 to 45 mas, but there is no ground-based parallax for comparison. GD 154 probably has a "very thin" hydrogen layer, but this is not proven. The seismological determination rests on the detection of three independent modes by Pfeiffer et al. (1996), which is oddly small compared to the number of modes present in other cool DAV pulsators, like G 29-38, HL Tau 76, and G 191-16 (Kleinman 1995). Nature is trying to tell us something here.

\subsection{Other DAV Stars}

Bradley (1993) discusses three other DAV stars: GD 385, PG 2303+242, and GD 66. I discuss these stars briefly in light of the theoretical and observational advances made since 1993.

\section{$6.8 .1 G D 385$}

GD 385 has only one independent mode at $256 \mathrm{~s}$ (Kepler 1984). This period is sufficiently long that assuming it is the $\ell=1, k=1$ mode places no constraint on the hydrogen layer mass. If one uses the identified modes of other DAV stars as a guide (Clemens 1994), then the $256 \mathrm{~s}$ mode would likely be the $\ell=1, k=3$ mode and the hydrogen layer mass would be about $10^{-4} \mathrm{M}_{\star}$. The weakness is that I have to assume a mode identification before I can determine the hydrogen layer mass. We either need an independent determination of the mode identity, to discover more observed modes, or prove the period relations of Clemens so that we can use it to derive the mode identity. 


\subsubsection{PG $2303+242$}

PG 2303+242 is one of the long period DAV stars with a complicated power spectrum (Vauclair et al. 1992). Given the difficulties that Kleinman had to find all the normal modes in G 29-38 and the inclusion of "combination" and harmonic peaks in the seismological analysis, the results of Bradley (1993) must be considered premature. A quick look at the periods that remain after throwing out the combination and harmonic peaks shows period spacings ranging from $10 \mathrm{~s}(463-453 \mathrm{~s})$ up to about $80 \mathrm{~s}$ (this could be about $40 \mathrm{~s}$ ). My present suspicion is that we may be seeing a combination of $\ell=1$ and 2 peaks, but we will need an observational effort similar to what Kleinman (1995) devoted to G 29-38 to reveal the true mode spectrum of this star. The mode structure appears to be rich enough that it is likely to be worth the effort.

\subsubsection{GD 66}

GD 66 is an object that has a moderate number of modes present, and apparently more stable amplitudes than the cooler pulsators. The three shortest periods (197, 271, and $301 \mathrm{~s}$ ) from Fontaine et al. (1985) are similar to those of G 117-B15A. The problem with this star is the mode identification; one has to make some drastic assumptions about the mode identity, which then effectively dictates the "derived" hydrogen layer mass. For instance, Bradley (1993) relies exclusively on trapped mode period relations to arrive at a hydrogen layer mass of $\mathrm{M}_{\mathrm{H}} \sim 2 \times 10^{-9} \mathrm{M}_{\star}$. This analysis presumes that trapped modes will be preferentially excited to higher amplitudes, and the hotter DAV stars don't seem to support this. Therefore, I could equally well use mode assignments based on Clemens's (1994) period templates, which would lead me to a hydrogen layer mass of about $10^{-4} \mathrm{M}_{\star}$. As a final alternative, I use the $11,980 \mathrm{~K}$ temperature and mass of $0.64 \mathrm{M}_{\odot}$ from Bergeron et al. (1995) and assume the $197 \mathrm{~s}$ mode is $\ell=1, k=1$. I find a $0.65 \mathrm{M}_{\odot}$ model with $10^{-6} \mathrm{M}_{\star}$ or $10^{-7} \mathrm{M}_{\star}$ will fit the three short period modes nicely at $12,000 \mathrm{~K}$. For instance, the $10^{-7} \mathrm{M}_{\star}$ model has the first four modes at $202,258,272$, and 302 s. Lest the reader think this fit is significant, remember that I have to assume a mode identification. If the $\ell=1$ identification is correct, then we have at least two likely hydrogen layer mass ranges for GD 66; otherwise, these are just two out of a large number of possible models for GD 66. Again, it will be 
useful to examine this object with single-site data and the WET to determine the observed period structure; this is a point I will return to later.

\section{Some Observations about DAV White Dwarf Seismology}

After a long struggle, we are finally beginning to attempt and make progress in the seismology of DAV white dwarfs. Seismology of the "hotter" DA white dwarfs is starting to bear fruit, especially with help from spectroscopic $\ell$ identifications. Even if one has to make assumptions, one can (at times) confine the allowed hydrogen layer mass to several narrow ranges, depending on the $k$ value assumed. The "cool" DA white dwarfs also show promise, but only when enough modes are present, a là G 29-38 and HL Tau 76.

While much is made of the difference between the hot and cool ZZ Ceti pulsators, there is another contrast that seems to have escaped attention. Two cool DAV stars (GD 154 and HS 0507+0434) seem to have only a few linearly independent modes in a "forest" of combination peaks and harmonics, while at the other extreme is G 29-38, with many modes along with the nonlinear peaks. Either this is an observational selection effect that needs to be revealed, or we actually have two extremes of cool ZZ Ceti pulsators. Fortunately, we will have a WET campaign on HS $0507+0434$ (Jordan et al. 1997) in the near future; we may also need long timebase single-site monitoring to fully reveal the seismological potential of HS $0507+0434$.

The sampling of DAV white dwarfs also reveal that ground-based parallaxes cannot be trusted too much. In particular, it is highly desirable to determine independent parallaxes for $\mathrm{G} 117-\mathrm{B} 15 \mathrm{~A}$ and $\mathrm{R} 548$, either with another parallax program or with a satellite that can determine the parallax of a 15th magnitude star.

\section{What DAV Stars Say About the Hydrogen Layer Mass}

At present, the seismological results do not give a clear answer to the "thick" versus "thin" debate. $A L L$ of the above stars (except G 29-38) could have hydrogen layers near $10^{-4} \mathrm{M}_{\star}$, which would validate Clemens' (1994) conclusion. In particular, G 226-29, L 192 , and GD 165 seem to require a thick hydrogen layer, and the fine structure splitting of R 548 favors one. However, the other stars have other possible hydrogen layer mass values that cannot be ruled out. 
My present verdict is that the seismological results are consistent with a range of hydrogen layer masses, but it is possible that many have thick hydrogen layers.

Even if we find that most DAV white dwarfs have thick hydrogen layers, we still have to fit it into the inferred hydrogen layer mass trends of Vennes et al. (1997) for the hottest DA white dwarfs and Bergeron etal $(1990,1997)$ for the coolest white dwarfs. As Fontaine \& Wesemael (1997) stress, there are two major channels for white dwarfs, and this does not include at least two other minor channels. I do not consider it surprising that the current picture appears to be unclear, and the old quote that "anything not forbidden is mandatory" is appropriate.

\section{Future Prospects}

The most pressing observational need we have is a way to determine mode identifications independently, especially for $k$ values. This will confine the bands of hydrogen layer masses (one for each mode ID assumption) to a single choice. Also, we would be able to independently confirm the mode identification Clemens arrived at using the "template" of DAV periods. If confirmed, then we will some interesting dilemmas concerning the spectral evolution of DA white dwarfs, especially those with massive $M_{H} \sim 10^{-4} M_{\star}$ hydrogen layers.

After we determine the internal structure of L 19-2, GD 165, and G 185-32, we will have only G 238-53 and GD 385 from the group of "simple" DAV stars that have escaped WET scrutiny. Neither is an immediate WET target nor do they deserve to be; G 238-53 needs more single-site data to determine just how rich the period structure is, and GD 385 has fairly extensive single-site data (Kepler 1984) that turned up a doublet at $256 \mathrm{~s}$ and a nonlinear combination peak at $128 \mathrm{~s}$. However, R 548 deserves a WET campaign to see if we can confirm the tentative detection of extra modes mentioned by Kepler (1995a) and also obtain enough data to refine the rates of period change from the values published by Tomaney (1987).

I consider BPM 37093 to be in a class by itself, because it is so massive $\left(\mathrm{M}_{\star} \approx 1.1 \mathrm{M}_{\odot}\right)$ that theory predicts must be at least $50 \%$ crystallized (see Montgomery 1997 and Kanaan 1997). This star is deservedly a WET target to search for the needed modes to see if we can observationally confirm one of the fundamental predictions of dense plasma physics. 
There is a group of intermediate-period DAV stars that so far is largely ignored. These stars are GD 66, GD 99, G 207-9, HS $0507+0434$, and KUV 11370+4222. Each has at least one or two modes in common with the hotter pulsators, and at the same time has a mode at 600 or more seconds. HS $0507+0434$ will soon be a WET target, mainly because of its double degenerate status. However, I believe that GD 66 and GD 99 would be good future WET targets, because both have a fair amount of published and/or unpublished data that shows promise.

Then we come to the cool DAV stars, and I place about 14 (half!) of the DAV stars in this category. These are demanding objects to study, because a WET run is not enough to decipher them. Kleinman's (1995) work on G 29-38 shows what is required. However, I see two major reasons to pick a few of these objects for more intensive study. First, we need to see if the cooler DAV stars have hydrogen layer masses similar to the hotter DAV stars. Recall that the Bergeron et al. (1990) observations suggest that there may be increasing amounts of helium in DA atmospheres just below the DAV instability strip; can we find any structural evidence in the cool DAV stars? Second, the hotter DAV stars seem to have hydrogen layer masses between $10^{-4} \mathrm{M}_{\star}$ and $10^{-7} \mathrm{M}_{\star}$; GD 154 on the other hand, has the favored hydrogen layer mass of $2 \times 10^{-10} \mathrm{M}_{\star}$. Also, G 29-38 may have a hydrogen layer mass of about $5 \times 10^{-7} \mathrm{M}_{\star}$. We need more cool DAV star hydrogen layer masses to see if we really have a difference or not. Finally, the cool DAV stars also show modes that cluster together in period space, suggesting that even these stars have something in common. For future WET targets, I suggest HL Tau 76 as the highest priority, followed by G 191-16, PG2303+242, and/or EC14012-1446.

Also, since 1993, five new ZZ Ceti stars were discovered, and a number of their periods fall into the cool DAV "template". Two have periods near $610 \mathrm{~s}$, which a very popular frequency amongst the cool DAV stars. EC 23487-2424 (Stobie et al. 1993) has 804 and 868 s periods, similar to two of the G $29-38$ periods. EC $14012-1446$ (Stobie et al. 1995) does even better, with three (out of five) periods that are within $6 \mathrm{~s}$ of periods found in G 29-38. The two obvious periods (494 and $618 \mathrm{~s}$ ) of KUV 08368+4026 (Vauclair et al. 1996) are similar to the 500 and $610 \mathrm{~s}$ periods of G 29-38. The periods of KUV $11370+4222$ and HS $0507+0434$ lie in between those of the hot and cool pulsators; some of the periods lie close to those of other DAV stars, while the 445 s period of HS $0507+0434$ appears to be unique. 
The fact that almost all the periods of G 29-38 (the best-studied cool DAV) has at least one duplicate is interesting. Periods near 400, 500, 610 , and $730 \mathrm{~s}$ seem to be especially favored. Most remarkable is the $610 \mathrm{~s}$ group, where 8 of $14 \mathrm{cool}$ DAVs have a mode. Further study is clearly warranted; even if only to show that the common periods are a remarkable coincidence.

I started my research on variable white dwarf stars 10 years ago. At that time, the DAV stars had a hodge-podge of known periods with no appearance of underlying order. Now we have at least tentative hydrogen layer mass determinations for seven of the 28 known DAV stars, and there is hope for even more soon. Also, Bradley (1997) made the first demonstration that core $\mathrm{C} / \mathrm{O}$ profiles can be constrained through seismology. The most serious hindrance to further progress is our desperate need for independent methods of identifying the $\ell$, and especially the $k$-values of observed modes. Success will allow us to more tightly constrain the seismological fits, and could also prove whether or not the common period trends presented by Clemens (1994) is the result of a common mode structure or a cruel coincidence. Finally, in spite of what the WET has done for DAV star seismology, I note that it is not always enough, particularly for the cool DAVs. What WET can do is provide a detailed "snapshot" that single-site data can complement to reveal the full set of linearly independent modes and the nonlinear interaction peaks. Given that half of the know DAV stars fall into what I call cool DAVs, we must eventually consider devoting the observational resources for them.

\section{References}

Bergeron, P., et al. 1993, AJ, 106, 1987

Bergeron, P., Ruiz, M.T., \& Leggett, S.K. 1997, ApJS, 108, 339

Bergeron, P., Wesemael, F., \& Fontaine, G. 1990, ApJ, 351, L21

Bergeron, P., Wesemael, F., Lamontagne, R., Fontaine, G., Saffer, R.A., \& Allard, N.F. 1995, ApJ, 449, 258

Bergeron, P., Saffer, R.A., \& Liebert, J. 1992, ApJ, 394, 228

Bradley, P.A. 1993, Ph. D. Thesis, Univ. Texas

Bradley, P.A. 1996, ApJ, 468, 350

Bradley, P.A. 1997, ApJ, submitted

Bradley, P.A., \& Kleinman, S.J. 1997, in White Dwarfs, Proc. of the 10th European Workshop on White Dwarf Stars, ed. J. Isern, E. GarciaBerro, \& M. Hernanz (Kluwer: Dordrecht), 445 
Brassard, P., Fontaine, G., Wesemael, F. \& Talon, A. 1993, in White Dwarfs: Advances in Observations and Theory, ed. M.A. Barstow (Dordrecht: Kluwer), 485

Brassard, P., \& Fontaine, G. 1994, in Proc. of IAU Colloq. 147, The Equation of State in Astrophysics, ed. G. Chabrier \& E. Schatzman, (Cambridge: Cambridge Univ.), 560

Clemens, J.C. 1994, Ph. D. Thesis, Univ. Texas

Dziembowski, W. 1977, Acta Astron., 27, 203

Fontaine, G., \& Brassard, P. 1994, in Proc. of IAU Colloq. 147, The Equation of State in Astrophysics, ed. G. Chabrier \& E. Schatzman, (Cambridge: Cambridge Univ.), 347

Fontaine, G., Brassard, P., \& Wesemael, F. 1994, ApJ, 428, L61

Fontaine, G., Brassard, P., Bergeron, P., \& Wesemael, F. 1992, ApJ, 399, L91

Fontaine, G., Brassard, P., Bergeron, P., \& Wesemael, F. 1996, ApJ, 469, 320

Fontaine, G., \& Wesemael, F. 1987, in IAU Colloq. 95, The Second Conference on Faint Blue Stars, ed. A.G.D. Philip, D.S. Hayes, \& J. Liebert, (Schenectady: Davis), 319

Fontaine, G., \& Wesemael, F. 1991, in IAU Symp. 145, The Photospheric Abundance Connection, ed. G. Michaud \& A. Tutukov, (Dordrecht: Reidel), 421

Fontaine, G., \& Wesemael, F. 1997, in White Dwarfs, Proc. of the 10th European Workshop on White Dwarf Stars, ed. J. Isern, E. GarciaBerro, \& M. Hernanz (Kluwer: Dordrecht), 173

Fontaine, G., Wesemael, F., Bergeron, P., Lacombe, P., Lamontagne, R., \& Saumon, D. 1985, ApJ, 294, 339

Jordan, S., Koester, D., Vauclair, G., Dolez, N., Heber, U., Reimers, D., Chevron, M., \& Dreizler, S. 1997, A\&A, in press

Kanaan, A. 1997, Baltic Astron., 6, in press

Kepler, S.O. 1984, ApJ, 278, 754

Kepler, S.O., 1997, in "A Half Century of Stellar Pulsation Interpretations:

A Tribute to Arthur N. Cox", ed. P.A. Bradley \& J.A. Guzik, ASP

Conf. Series, in press.

Kepler, S.O., \& Bradley, P.A. 1995, Baltic Astron., 4, 166

Kepler, S.O., et al. 1995a, Baltic Astron., 4, 238

Kepler, S.O., et al. 1995b, Baltic Astron., 4, 221

Kepler, S.O., et al. 1995c, ApJ, 447, 874

Kepler, S.O., et al. 1995d, Baltic Astron., 4, 302

Kleinman, S.J. 1995, Ph. D. Thesis, Univ. Texas 
Koester, D., \& Vauclair, G. 1997, in White Dwarfs, Proc. of the 10th European Workshop on White Dwarf Stars, ed. J. Isern, E. GarciaBerro, \& M. Hernanz (Kluwer: Dordrecht), 429

Mazzitelli, I., \& D'Antona, F. 1986a, ApJ, 308, 706

Mazzitelli, I., \& D'Antona, F. 1986b, ApJ, 311, 762

Montgomery, M. 1997, Baltic Astron., 6, in press

O'Donoghue, D., \& Warner, B. 1982, MNRAS, 200, 573

O'Donoghue, D., \& Warner, B. 1987, MNRAS, 228, 949

Pfeiffer, B., et al., 1996, A\&A, 314, 182

Robinson, E.L., Mailloux, T.M., Zhang, E., Koester, D., Steining, R.F., Bless, R.C., Percival, J.W., Taylor, M.J., \& van Citters, G.W. 1995, ApJ, 438, 908

Salaris, M., Dominguez, I., Garcia-Berro, E., Hernanz, M., Isern, J., \& Mochkovitch, R. 1997, ApJ, 486, 413

Stobie, R.S., Chen, A., O'Donoghue, D., \& Kilkenny, D. 1993, MNRAS, 263, L13.

Stobie, R.S., O'Donoghue, D., Ashley, R., Koen, C., Chen, A., \& Kilkenny, D., 1995, MNRAS, 272, L21

Sullivan, D.J., 1995 Baltic Astron., 4, 261

Tomaney, F. 1987, in IAU Colloq. 95, The Second Conference on Faint Blue Stars, ed. A.G.D. Philip, D.S. Hayes, \& J. Liebert, (Schenectady: Davis), 673

Turon, C., \& van Leeuwen, F. 1995, Astrophysical Applications of Stellar Pulsation, ed. R.S. Stobie \& P.A. Whitelock, ASP Conf. Series, 83, 251

van Altena, W.F., Lee, J.T., \& Hoffleit, E.D. 1994, The General Catalogue of Trigonometric Parallaxes (New Haven: Yale University Observatory)

Vauclair, G., Belmonte, J.A., Pfeiffer, B., Grauer, A.D., Jiménez, A., Chevreton, M., Dolez, N., Vidal, I., \& Herpe, G. 1992, A\&A, 264, 547

Vauclair, G., Schmidt, H., Koester, D., \& Allard, N. 1997, A\&A, in press Vennes, S., Thejl, P.A., Galvan, R.G., \& Dupuis, J. 1997, ApJ, 480, 714

Winget, D.E., et al. 1994, ApJ, 430, 839

Wood, M.A. 1992, ApJ, 386, 539

Wood, M.A. 1994, in Proc. of IAU Colloq. 147, The Equation of State in Astrophysics, ed. G. Chabrier \& E. Schatzman, (Cambridge: Cambridge Univ.), 612

Wood, M.A. 1995, Proc. 9th European Workshop on White Dwarf Stars, eds. D. Koester \& K. Werner (Berlin: Springer), 41 


\section{M98003016}

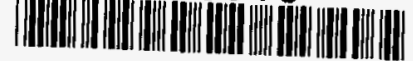

Report Number (14) $L A-U R--97-4011$

CONF-9707147=-

دubl. Date (11)

Sponsor Code (18)

199710

JC Category (19)

NASA, XF

UC-OOO, DOE/ER 